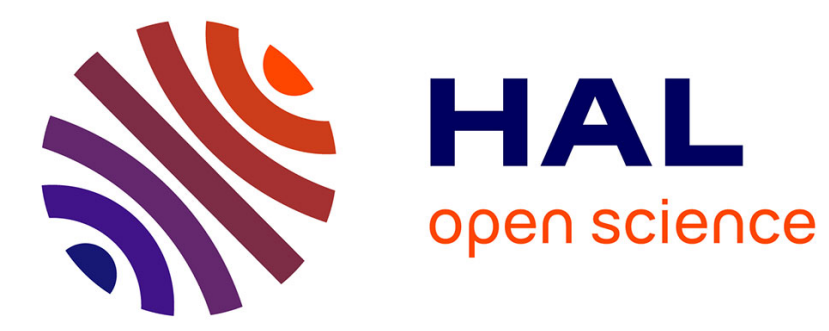

\title{
Finite Larmor Radius Effects in Two-Dimensional Electrostatic Plasma Turbulence
}

\author{
B. Krane, H. Pécseli, K. Rypdal, J. Trulsen
}

\section{To cite this version:}

B. Krane, H. Pécseli, K. Rypdal, J. Trulsen. Finite Larmor Radius Effects in Two-Dimensional Electrostatic Plasma Turbulence. Journal de Physique IV Proceedings, 1995, 05 (C6), pp.C6-31-C636. 10.1051/jp4:1995606 . jpa-00253969

\section{HAL Id: jpa-00253969 https://hal.science/jpa-00253969}

Submitted on 1 Jan 1995

HAL is a multi-disciplinary open access archive for the deposit and dissemination of scientific research documents, whether they are published or not. The documents may come from teaching and research institutions in France or abroad, or from public or private research centers.
L'archive ouverte pluridisciplinaire HAL, est destinée au dépôt et à la diffusion de documents scientifiques de niveau recherche, publiés ou non, émanant des établissements d'enseignement et de recherche français ou étrangers, des laboratoires publics ou privés. 


\title{
Finite Larmor Radius Effects in Two-Dimensional Electrostatic Plasma Turbulence
}

\author{
B. Krane, H.L. Pécseli*, K. Rypdal** and J. Trulsen*** \\ University of Troms $\phi, 9037$ Troms $\phi$, Norway \\ * University of Oslo, Institute of Physics, Box 1048 Blindern, 0316 Oslo, Norway \\ ** University of Troms $\phi, 9037$ Troms $\emptyset$, Norway \\ *** University of $O$ slo, Institute of Theoretical Astrophysics, Box 1029 Blindern, \\ 0315 Oslo, Norway
}

\begin{abstract}
Low frequency electrostatic turbulence in strongly magnetized, low $\beta$-plasmas, is studied in two spatial dimensions. In this limit the guiding center velocity in the direction perpendicular to a homogeneous magnetic field is approximated by the $\mathbf{E} \times \mathbf{B}_{0} / B_{0}^{2}$-velocity. The electron Larmor radius can safely be set to zero for most relevant conditions, but the ion-dynamics are noticeably influenced by finite Larmor radius effects. We analyzed the effects of finite Larmor radius corrections on the ion velocity and density by using a fluid model where these effects are included by a computationally simple filtering operation. The equilibrium spectra are investigated and compared with analytical results. The consequences of finite Larmor radius corrections for the turbulent diffusion of charged particles across magnetic field lines are studied by obtaining the Lagrangian correlation functions for passive test particles convected by the plasma flow.
\end{abstract}

\section{Introduction}

Low frequency electrostatic turbulence in strongly magnetized plasmas allow the analysis to be carried out in two dimensions in the limit where magnetic field lines can be considered equipotential, and the magnetic field assumed homogeneous. Then the local plasma velocity can be approximated by $\mathrm{v}=-\nabla \Phi \times \mathbf{B}_{\mathbf{0}} / B_{0}^{2}$ provided characteristic frequencies are well below the ion gyro frequency, $\Omega_{c i}$. The equation of continuity for electrons and ions then becomes the Euler equation

$$
\frac{\partial n_{e, i}(\mathbf{r}, t)}{\partial t}+\frac{1}{B_{0}}\left[\Phi(\mathbf{r}, t), n_{e, i}(\mathbf{r}, t)\right]=0
$$

where [,] denotes Poisson brackets and the electrostatic potential $\Phi$ takes the role of the stream function. Together with Poisson's equation

$$
\nabla^{2} \Phi(\mathbf{r}, t)=\frac{e}{\varepsilon_{0}}\left(n_{i}(\mathbf{r}, t)-n_{e}(\mathbf{r}, t)\right)
$$

a closed, standard, equation is obtained for $\Phi$. Polarization drifts are ignored and it is assumed that the perturbations are strictly flute like i.e. B-field aligned, so that electrons can not maintain a Boltzmann equilibrium by flowing along magnetic field lines. This model have been extensively investigated by Seyler et al. [1]. 
Equation (1) assumes that the positions of the charged particles are well approximated by their guiding-center. For electrons this is valid for most physically relevant cases, but this assumption might fail for the ions due to their finite Larmor radii. A straight forward, but computationally costly, remedy assurnes a full gyrating particle description. A simpler fluid model includes the lowest order finite Larmor radius (FLR) effects by introducing an operator acting on the electric field [2]. By this the averaging of the electric field along the circular gyro-orbit of an ion with gyro-radius $r_{L}$ is approximated, with the assumption that the characteristic length scale for the electric field variations is much larger than $r_{L}$. The resulting ion-gyrocenter velocity is obtained as

$$
\left(1+\frac{1}{4} r_{L}^{2} \nabla^{2}\right) \mathbf{E} \times \mathbf{B}_{\mathbf{0}} / B_{0}^{2} .
$$

When polarization drifts are ignored this approximation is formally adequate, but is numerically unfavorable by incorrectly weighting short scale length fluctuations. Even if their amplitude is by assumption small, this weighting is numerically unfavorable. An alternative and numerically more robust model was suggested by Knorr et al. [3] where a fluid model is retained but the averaging along the ion orbit is retained accurate to all orders. The resulting numerical scheme is easy to implement and is only slightly more time consuming than the solution of (3). Analytical results are available for the model which can serve as nontrivial tests of the code.

\subsection{FLR Corrections to Arbitrary Order}

A computationally preferable alternative to (3) is to retain the full expression from the averaging of the electric field over the gyro orbit. As mentioned earlier, the electric field experienced by a gyrating charged particle is no longer the same as the electric field evaluated at the guiding center. Knorr et al. [3] derived an expression for the corrected guiding center velocity, indicated by an overbar

$$
\overline{\mathbf{v}}(\mathbf{x})=\sum_{\mathbf{k}} J_{0}\left(k r_{L}\right) \mathbf{v}_{\mathbf{k}} \exp (i \mathbf{k} \cdot \mathbf{x})
$$

where the vector-component $\mathbf{v}_{\mathbf{k}}$, without the overbar, is obtained from the $\mathbf{E} \times \mathbf{B}$-velocity evaluated at the guiding center and $J_{0}$ is the Bessel function of order zero.

A series expansion of $J_{0}\left(k r_{L}\right)$, assuming $k r_{L} \ll 1$ gives $J_{0}\left(k r_{L}\right) \approx 1-\frac{1}{4}\left(k r_{L}\right)^{2}$. Retaining only the leading terms, we obtain the same correction to the guiding center velocity as found by Chen [2], equation (3). From the series expansion we also note that the FLR correction corresponds to a reduction in the guiding center velocity. In the formal limit where $r_{\mathrm{L}}$ ultimately reaches infinity we have $J_{0}(\infty)=0$ and the ion density turns into a uniform background, decoupled from the dynamics. Mathematically, this situation turns out to be equivalent to the zero Larmor radius case where the equations describing the electron and ion density could be combined, leading to a single equation for the potential. This limit of $r_{\mathrm{L}} \rightarrow \infty$ is, however, inconsistent with the assumptions in the derivations.

The effective, or corrected, guiding center velocity discussed in this section is distinct from the velocity appearing in the usual fluid equations; in that case the velocity is the vector sum of all particle velocities crossing a small reference area.

Also the ion density has to be corrected for FLR-effects. Due to the rapid gyration of the charged particles with respect to their guiding center, their charge can be considered as being deposited on a ring with radius $r_{\mathrm{I}}$ around the guiding center.

The analysis is very similar to that giving the corrections to the $\mathbf{E} \times \mathbf{B}$-velocity and the result is

$$
\bar{n}(\mathbf{x})=\sum_{\mathbf{k}} J_{0}\left(k r_{\mathrm{L}}\right) n_{\mathrm{k}} \exp (i \mathbf{k} \cdot \mathbf{x}) .
$$

From a prescribed guiding center density $n(\mathbf{x})$ we are now able to calculate the corresponding charge density by filtering the Fourier coefficients of $n(\mathbf{x})$ with $g_{\mathbf{k}}=J_{0}\left(k r_{\mathrm{L}}\right)$, or alternatively by performing the appropriate convolution in configuration space. 
Physically, the inclusion of FLR-effects consists of the following steps; first the effective electric field is determined, corresponding to the appropriate Larmor radius for the particle. Then the particle guiding cents move in response to this effective field with the density of the appropriate guiding centers follow a continuity equation. The electric field which enters this derivation is obtained from Poisson's equation, where the local electron and ion densities are obtained for the given guiding center density by distributing the particles along their circular orbit as described before.

In dimensionless units the governing equations, with the FLR-corrections incorporated, take the form

$$
\begin{aligned}
\frac{\partial n_{i}}{\partial t}+\nabla \cdot\left(n_{i} \mathbf{\mathbf { v }}\right) & =0 \\
\frac{\partial n_{e}}{\partial t}+\nabla \cdot\left(n_{e} \mathbf{v}\right) & =0 \\
\mathbf{v} & =-\nabla \Phi \times \mathbf{b} \\
\nabla^{2} \Phi & =-\left(\bar{n}_{i}-n_{e}\right)
\end{aligned}
$$

and where $\overline{\mathrm{v}}$ and $\bar{n}$ are obtained from equation 4 and 5 , respectively. Again it should be stressed that $n_{e}$ and $n_{i}$ is the electron and ion guiding center densities, respectively.

The system of equations is solved on a square grid with length $L=10$, and periodic boundary conditions. We used a third order Adams-Bashforth predictor-corrector method to advance the solution in time. To calculate the spatial operators we used a Fourier collocation method which consist of calculating all derivatives in Fourier space before transforming back to configuration space where the nonlinear products are evaluated. Hence, the Fourier coefficients required for the FLR corrections, equation (4) and (5), are available without additional computational expense.

First we demonstrate the results from a simulation where all the ions are assumed to have the same Larmor radius. Next, we include several ion components, with different Larmor radii, which interact through the collective electric field.

\section{Equilibrium spectra}

With the FLR corrections given by the foregoing arguments, Knorr and Pécseli worked out an analytical expression for the spectral equilibrium distribution [4]. We use these results as a test of our program, but as they have not been tested before the results are interesting by themselves.

In Fourier space the system of equations (6)-(9) is represented by their truncated Fourier series. It is now easily shown that this truncated system has three invariants. These invariants, denoted by $\Omega_{e}, \Omega_{i}$ and $\mathcal{E}$, are conserved even for a finite number of Fourier modes [4]:

$$
\Omega_{e, i}=\sum_{k_{\mathbf{0}}}^{k_{\max }}\left|n_{e, i \mathbf{k}}\right|^{2}, \quad \mathcal{E}=\sum_{\mathrm{k}_{\mathbf{0}}}^{k_{\max }}\left|\bar{n}_{i \mathbf{k}}-n_{e \mathbf{k}}\right|^{2} / k^{2} .
$$

By considering a micro-canonical ensemble of systems consisting of the Fourier coefficients of $n_{e}$ and $n_{i}$, evolving under the restriction that the three invariants must be conserved, the equilibrium is given by the Boltzmann-Gibbs distribution. This distribution is characterized by the inverse temperatures $\mu_{e}, \mu_{i}$ and $\beta$. By differentiating the logarithm of the partition function with respect to these temperatures, various expectation values are found, i.e. the spectral energy distribution

$$
\left\langle\mathcal{E}_{\mathbf{k}}\right\rangle=\frac{1}{\beta+c_{\mathbf{k}}^{2} k^{2}} \quad \text { with } \quad c_{\mathbf{k}}^{2}=\frac{1}{\mu_{e}^{-1}+g_{\mathbf{k}}^{2} \mu_{i}^{-1}},
$$

and the spectral distribution of the electron and ion guiding center density

$$
\left\langle\left|n_{e \mathbf{k}}\right|^{2}\right\rangle=\mu_{e}^{-1}\left(1-\frac{\beta c_{k}^{2} \mu_{e}^{-1}}{\beta+c_{k}^{2} k^{2}}\right), \quad\left\langle\left|n_{i \mathbf{k}}\right|^{2}\right\rangle=\mu_{i}^{-1}\left(1-\frac{\beta c_{k}^{2} g_{k}^{2} \mu_{i}^{-1}}{\beta+c_{k}^{2} k^{2}}\right) .
$$


With $\mu_{e}=1.0, \mu_{i}=0.1$ and $\beta=-9 \times 10^{-3}$ these expectation values are used as initial conditions for the simulations. Note that no singularity arises in the spectrum, for small negative $\beta$-values, because only discrete wavenumbers enters the analysis. The spectrum is positive definite for all $|\mathbf{k}|>0$, and $|\mathbf{k}|=0$ does not enter the dynamic evolution.

In figure 1 , the time averaged, numerically obtained, spectral distribution is compared with the theoretical result for two different Larmor radii.
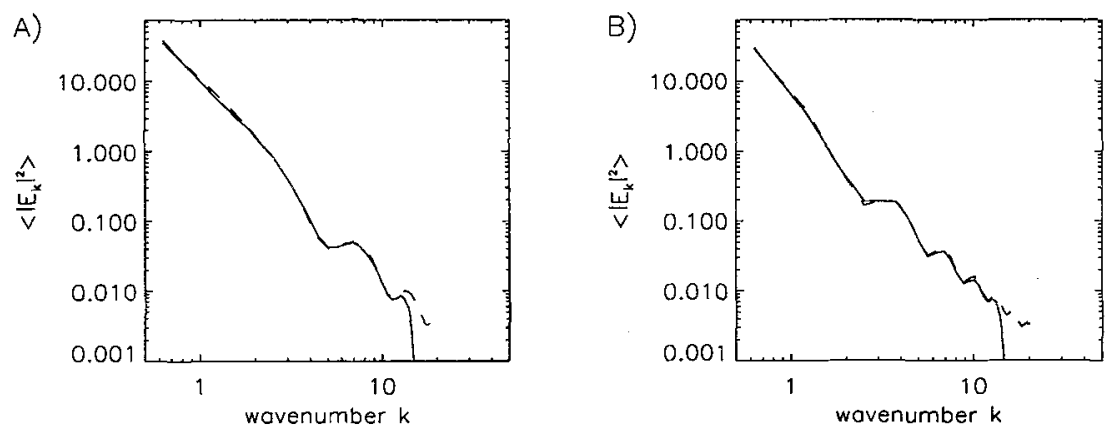

Figure. 1: Log-log plots of the time averaged mumerically calculated spectrum, solid line, compared to the analytical result given by a dashed line, for $r_{L}=0.5$ and $r_{L}=1.0$.

The agreement between the numerical results and the theoretical prediction is good, indicating that the results quoted above represent the correct wavenumber spectral distributions. In particular, it can be argued that $\Omega_{e}, \Omega_{i}$ and $\mathcal{E}$ are the only invariants of the truncated system (6)-(9).

The stability of the spectra was explicitly verified by demonstrating the relaxation of a perturbed spectrum towards the theoretical distribution of energy in wavenumber space.

\section{Turbulent diffusion}

We have used the code discussed in the foregoing section to analyze the consequences of the FLRcorrection for turbulent transport. In particular, it is demonstrated that different ion components, i.e. ions with different Larmor radii, diffuse at different rates. The most important quantity is the normalized Lagrangian correlation function, $R(s)=\langle\mathbf{v}(\mathbf{r}(t)) \cdot \mathbf{v}(\mathbf{r}(t+s))\rangle /\left\langle v^{2}\right\rangle$, which, for homogeneous and time stationary turbulence, contains all relevant information for obtaining the mean-square particle displacement, $\left\langle r^{2}(t)\right\rangle$. Thus, it is a well known result that

$$
\left\langle r^{2}\right\rangle=2\left\langle v^{2}\right\rangle t \int_{0}^{t}(1-s / t) R_{L}(s) d s .
$$

For large times, $t \gg \tau$, the diffusion limit, this gives $\left\langle r^{2}\right\rangle \approx 2\left\langle v^{2}\right\rangle \tau t$ where $\tau \equiv \int_{0}^{\infty} R_{L}(s) d s$ is the Lagrangian integral time scale. In the convection limit of small $t$ where the approximation $R_{L}(s) \simeq 1$ can be used, the expression (10) gives $\left\langle r^{2}\right\rangle \approx\left\langle v^{2}\right\rangle t^{2}$. In figure 2 we show numerically obtained Lagrangian correlation functions for two different simulations containing electrons with zero Larmor radius and one ion specie with the FLR-corrections taken into account.

As discussed in the foregoing section, the electric field power spectrum changes with the ion Larmor radii. A comparison between the electron and ion correlation functions corresponding to the same flow clearly indicates an increase in the integral time scale for the ions for increasing $r_{L}$. 

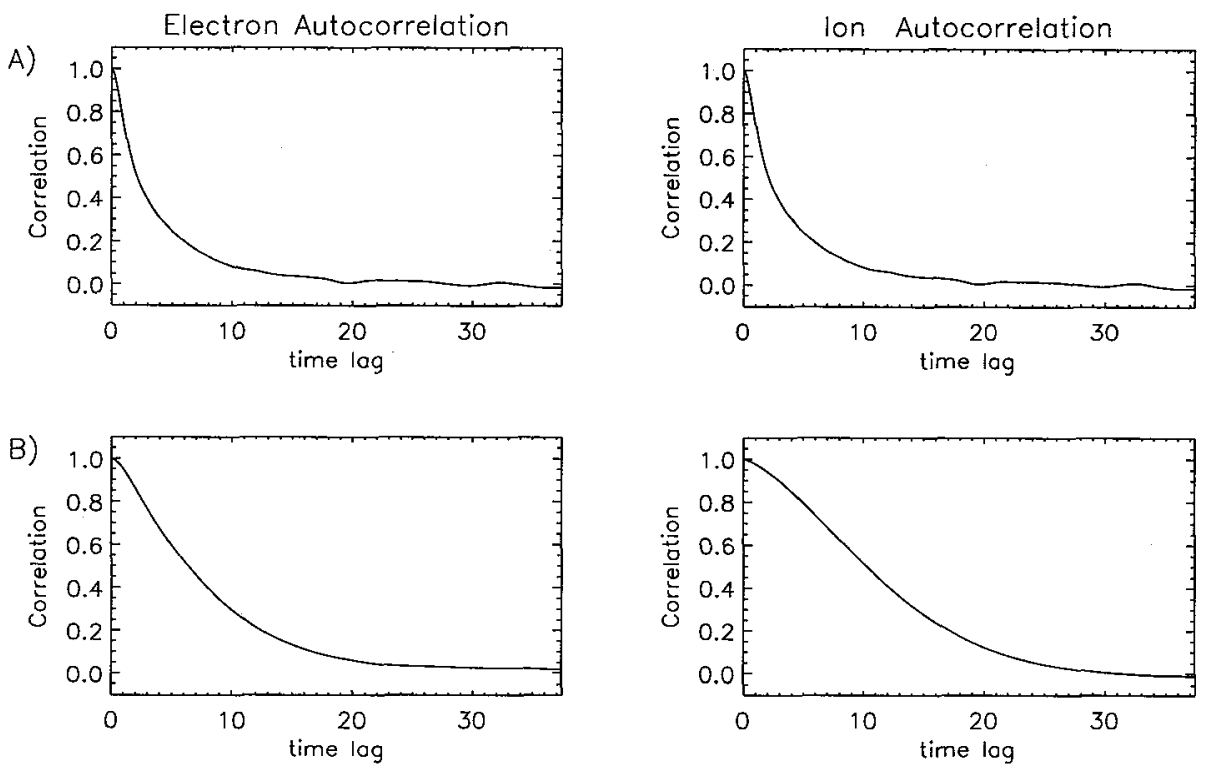

Figure. 2: Lagrangian velocity autocorrelation functions for electrons and ions obtained from simulations with one ion specie in addition to the electrons. The results are shown for two different ion Larmor radii, A) $r_{L}=0$ and B) $r_{L}=1$.

Results are shown in figure 2 for $r_{L}=0$, where electrons and ions have the same velocity statistics, and for $r_{L}=1$. The results are readily understood by the difference in rms-velocities for electrons, $\left\langle v_{e}^{2}\right\rangle=\int_{0}^{\infty} \mathcal{E}(k) d^{2} k$, and ions, $\left\langle v_{i}^{2}\right\rangle=\int_{0}^{\infty} \mathcal{E}(k) J_{0}^{2}\left(k r_{L}\right) d^{2} k$. Evidently $\left\langle v_{e}^{2}\right\rangle \geq\left\langle v_{i}^{2}\right\rangle$ since $J_{0}^{2}\left(k r_{L}\right) \leq 1$. The integral time scale can be estimated by the ratio of a length scale which is determined by $\mathcal{E}(k)$ and a velocity $\left\langle v^{2}\right\rangle^{1 / 2}$. Since ions and electrons move in the same electric field spectrum, the characteristic length is the same for both species, while their rms-velocities are different for different $r_{L}$ by the arguments given before. Correspondingly, we expect by this heuristic argument that the integral time scale increases for increasing $r_{L}$.

The system of equations (6)-(9) is easily extended to include the more realistic case where many different ion Larmor radii are simultaneously present in the flow. In figure 3 we show Lagrangian correlation functions for the case where 4 ion components with different Larmor radii are present in the same flow. Again we observe an increase in the integral time scale $\tau$ for increasing $r_{x}$.

\section{Conclusions}

In this paper we presented a computationally advantageous code for including finite Larmor radius effects in a fluid model. The problem was first studied numerically by Knorr et al [3], but in a formulation where they averaged over Larmor radii for ions having gyrocenters in a small area of configuration space. Physically, this averaging, which leads to a particularly simple form of the convolution, assumes that all the ions in that small area follow essentially the same orbit. This can be achieved if there is a strong collisional interaction between the ions, which enters as an additional assumption. The simplicity of the formalism used by Knorr et al. was thus obtained on the expense of a somewhat uncertain physical model, which we avoided in the present study. 


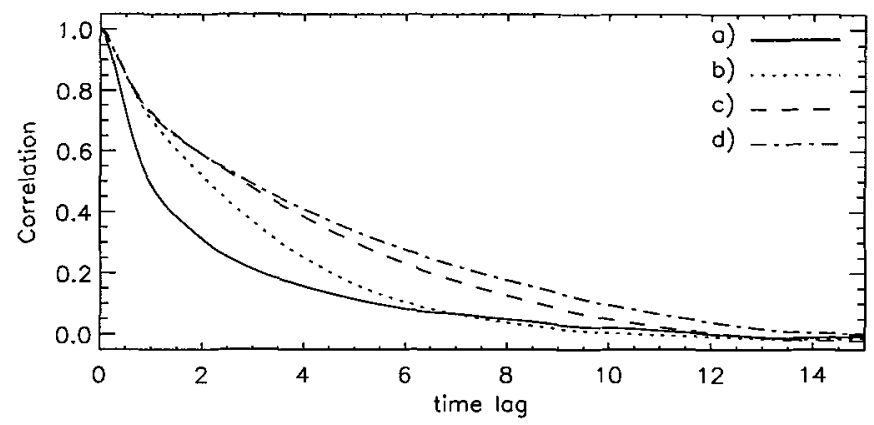

Tigure. 3: Lagrangian velocity autocorrelation functions for a simulation containing electrons and 4 ion species with different Larmor radii, $r_{L}=0,0.5,1$ and 1.5 .

In addition to standard accuracy tests, our code was tested by comparing numerically obtained equilibrium spectra with analytical results. Excellent agreement was found, adding confidence not only to our code but also to the theoretical analysis. These equilibrium spectra have not been analyzed before. We applied the code to a study of turbulent transport, demonstrating that FLReffects have a nontrivial effect on the dispersion of charged particles in magnetized plasmas. For turbulent, inhomogeneous plasmas this difference in diffusion rates will have significant influence on the build-up of ambipolar DC-electric fields, and subsequently also on the bulk flow characteristics.

We have generalized our model and numerical code to the case where the electrons can be assumed to have obtained a local isothermal Boltzmann equilibrium, while a two dimensional description is retained for the ions. These results will be presented in a different context.

\section{Acknowledgments}

This work was supported in part by the Norwegian Research Council for Science and Humanities and the program for supercomputing. One of us (B.K.) thanks "Tungregneutvalget" at NFR for providing computational time on the IBM-cluster in Oslo.

\section{References}

[1] C. E. Seyler, Y. Salu, D. Montgomery, and G. Knorr. Phys. Fluids 18 (1975) pp. 803-813.

[2] F. F. Chen. Introduction to Plasma Physics and Controlled Fusion. Plenum Press New York second edition (1990) pp. 36-38.

[3] G. Knorr, F. R. Hansen, J. P. Lynov, H. L. Pécseli, and J. Juul Rasmussen. Physica Scripta 38 (1988) pp. 829-834.

[4] G. Knorr and H. L. Pécseli. J. Plasma Phys. 41 (1988) pp. 157-170. 